\title{
NECESSARY AND SUFFICIENT CONDITIONS FOR OSCILLATIONS OF HIGHER ORDER DELAY DIFFERENTIAL EQUATIONS
}

\author{
$\mathrm{BY}$
}

\author{
G. LADAS, Y. G. SFICAS AND I. P. STAVROULAKIS
}

ABStRACT. Consider the $n$th order delay differential equation

$$
x^{(n)}(t)+(-1)^{n+1} \sum_{i=0}^{k} p_{i} x\left(t-\tau_{i}\right)=0, \quad t \geq t_{0},
$$

where the coefficients and the delays are constants such that $0=\tau_{0}<\tau_{1}<$ $\cdots<\tau_{k} ; p_{0} \geq 0, p_{i}>0, i=1,2, \ldots, k ; k \geq 1$ and $n \geq 1$. The characteristic equation of (1) is

$$
\lambda^{n}+(-1)^{n+1} \sum_{i=0}^{k} p_{i} e^{-\lambda \tau_{i}}=0 .
$$

We prove the following theorem.

THEOREM.(i) For $n$ odd every solution of (1) oscillates if and only if (2) has no real roots.

(ii) For $n$ even every bounded solution of (1) oscillates if and only if (2) has no real roots in $(-\infty, 0]$.

The above results have straightforward extensions for advanced differential equations.

1. Introduction. The oscillation theory of delay differential equations has been extensively developed during the past few years. See, for example, $[\mathbf{1}-\mathbf{1 0}]$ and the references cited therein. Most of the known results, however, give sufficient conditions for oscillations.

Our aim in this paper is to obtain necessary and sufficient conditions under which all solutions of the $n$th order delay differential equation

$$
x^{(n)}(t)+(-1)^{n+1} \sum_{i=0}^{k} p_{i} x\left(t-\tau_{i}\right)=0, \quad t \geq t_{0},
$$

oscillate when $n$ is odd, and necessary and sufficient conditions under which all bounded solutions oscillate when $n$ is even. The coefficients and the delays of the differential equation are assumed to be constants such that $0=\tau_{0}<\tau_{1}<\cdots<$ $\tau_{k} ; p_{0} \geq 0, p_{i}>0$ for $i=1,2, \ldots, k$ with $k \geq 1$ and $n \geq 1$.

As is customary, a solution is said to oscillate if it has arbitrarily large zeros. Solutions of $(1)$ are continuous functions $x$ defined on $\left[-\tau_{k}, \infty\right)$ that satisfy (1). As usual, we shall use the term "eventually" to mean "for sufficienty large $t$ ".

Received by the editors May 30, 1983. The results of this paper have been presented to the International Conference on Differential Equations held at the University of Alabama in Birmingham, March 21-26, 1983.

1980 Mathematics Subject Classification. Primary 34K15.

Key words and phrases. Delay differential equations, advanced arguments, oscillations.

(C) 1984 American Mathematical Society $0002-9947 / 84 \$ 1.00+\$ .25$ per page 
The characteristic equation of $(1)$ is

$$
F(\lambda) \equiv \lambda^{n}+(-1)^{n+1} \sum_{i=0}^{k} p_{i} e^{-\lambda \tau_{i}}=0 .
$$

The main result is the following.

THEOREM 1. (i) For $n$ odd all solutions of (1) oscillate if and only if (2) has no real roots.

(ii) For $n$ even all bounded solutions of (1) oscillate if and only if (2) has no real roots in $(-\infty, 0]$.

It should be noted that for $n$ even, $F(0) F(+\infty)<0$, so (2) always has positive roots. That is, (1) always has unbounded nonoscillatory solutions. However, the bounded solutions of (1) may or may not oscillate. For example, $x^{\prime \prime}(t)-x(t-\pi)$ $=0$ has the bounded oscillatory solution $x(t)=\sin t$, while the equation $x^{\prime \prime}(t)$ $-x(t-1 / \sqrt{e})=0$ has the bounded nonoscillatory solution $x(t)=e^{-t \sqrt{e}}$.

2. Proof of the main result. The following lemma will enable us to restate Theorem 1 in such a way that the odd and even cases of $n$ are proved simultaneously.

LEMMA 1. For $n$ odd (1) has no unbounded nonoscillatory solutions and (2) has no real roots in $[0, \infty)$.

ProOF. Clearly, (2) has no real roots in $[0, \infty)$. Next assume, for the sake of contradiction, that (1) has an unbounded nonoscillatory solution $x(t)$. Without loss of generality we may (and do) assume that $x(t)$ is eventually positive. Then, for $t_{1}$ sufficiently large,

$$
x^{(n)}(t)<0, \quad x^{(n-1)}(t)>0, \quad \text { and } \quad x^{\prime}(t)>0 \quad \text { for } t \geq t_{1} .
$$

Integrating (1) over $\left[t_{1}, t\right]$ we find

$$
x^{(n-1)}(t)-x^{(n-1)}\left(t_{1}\right)+\sum_{i=0}^{k} p_{i} \int_{t_{1}}^{t} x\left(s-\tau_{i}\right) d s=0 .
$$

Deleting some positive terms and using the fact that $x(t)$ is increasing, we obtain

$$
-x^{(n-1)}\left(t_{1}\right)+p_{1} x\left(t_{1}-\tau_{1}\right)\left(t-t_{1}\right)<0,
$$

which, as $t \rightarrow \infty$, leads to a contradiction.

In view of Lemma 1 , for $n$ odd every unbounded solution of (1) is oscillatory. Hence it suffices to prove part (i) of Theorem 1 only for bounded solutions. Therefore, the proof of Theorem 1 has been reduced to proving the following result for arbitrary $n$.

THEOREM 2. The following statements are equivalent.

(a) Every bounded solution of (1) oscillates.

(b) Equation (2) has no real roots in $(-\infty, 0]$.

PROOF. $(a) \Rightarrow(b)$. Otherwise (2) has a real root $\lambda_{0} \leq 0$ and so (1) has the bounded nonoscillatory solution $x(t)=e^{\lambda_{0} t}$. Contradiction.

(b) $\Rightarrow(\mathrm{a})$. (We prove it by contradiction.) Otherwise (1) has an eventually positive bounded solution $x(t)$. The rest of the proof will be divided into a series of six lemmas. Throughout the remainder of this paper $x(t)$ stands for the positive solution whose existence we have just assumed. 
LEMMA 2. Under the hypothesis that equation (2) has no roots in $(-\infty, 0]$, the number

$$
m=\min _{\lambda \geq 0}\left(\sum_{i=0}^{k} p_{i} e^{\lambda \tau_{i}}-\lambda^{n}\right)
$$

exists and is a positive constant.

LEMMA 3. For $i=0,1,2, \ldots, n$,

$$
(-1)^{i} x^{(i)}(t)>0, \quad \text { eventually. }
$$

LEMMA 4. There is a constant $B>1$ such that

$$
x\left(t-\tau_{k}\right)<B x(t), \quad \text { eventually }
$$

LEMMA 5. For any $\lambda \geq(B-1) / \tau_{k}$, where $B$ is as defined in Lemma 4,

$$
x^{\prime}(t)+\lambda x(t)>0
$$

and therefore

$$
\lim _{t \rightarrow \infty}\left[x(t) e^{\lambda t}\right] \neq 0 .
$$

LEMMA 6. Define $\lambda_{0}=p_{1}^{1 / n}, x_{0}(t)=x(t)$, and for each $j=1,2, \ldots$ set

$$
\lambda_{j}=\left(\lambda_{j-1}^{n}+m\right)^{1 / n}
$$

and

$$
x_{j}(t)=\sum_{i=0}^{n-1} \lambda_{j-1}^{n-1-i}(-1)^{i} x_{j-1}^{(i)}(t) .
$$

Then the following statements hold for each $j=0,1,2, \ldots$

(i) $x_{j}(t)$ is an eventually positive solution of (1) such that

$$
(-1)^{i} x_{j}^{(i)}(t)>0 \quad \text { for } i=0,1,2, \ldots, n \text { eventually. }
$$

(ii) There exists a positive constant $B_{j}$ such that

$$
x_{j}\left(t-\tau_{k}\right)<B_{j} x_{j}(t), \text { eventually. }
$$

(iii)

$$
(-1)^{n+1} x_{j}^{(n)}(t)+\lambda_{j}^{n} x_{j}(t)=x_{j+1}^{\prime}(t)+\lambda_{j} x_{j+1}(t)<0 .
$$

LEMMA 7. With the notation of Lemma 6 and under the hypothesis that equation (2) has no roots in $(-\infty, 0]$, the following statements are true for each $j=$ $0,1,2, \ldots$.

(i)

$$
\lim _{t \rightarrow \infty}\left[x_{j}^{(i)}(t) e^{\lambda_{j} t}\right]=0 \quad \text { for } i=0,1,2, \ldots
$$

(ii)

$$
\lim _{t \rightarrow \infty}\left[x(t) e^{\lambda_{j} t}\right]=0 .
$$


The desired contradiction in the proof of Theorem 2 follows from (7) and (14) by choosing $\lambda_{j} \geq \lambda$, that is, by choosing $j$ such that

$$
\left(p_{1}+j m\right)^{1 / n} \geq(B-1) / \tau_{k} .
$$

In the case $n=1$, Lemma 7 is unnecessary because (6) and (12) are contradictory for $\lambda_{j} \geq \lambda$. In fact, for $n=1$,

$$
x_{j}(t)=x(t) \text { for all } j
$$

and the last inequality in (12) reduces to

$$
x^{\prime}(t)+\lambda x(t)<0 .
$$

Proof of Lemma 2. Since $F(\lambda)=0$ has no negative root, $F(-\lambda)$ has no positive root. But

$$
\begin{aligned}
F(-\lambda) & =(-1)^{n} \lambda^{n}+(-1)^{n+1} \sum_{i=0}^{k} p_{i} e^{\lambda \tau_{i}} \\
& =(-1)^{n+1}\left(\sum_{i=0}^{k} p_{i} e^{\lambda \tau_{i}}-\lambda^{n}\right)=0 .
\end{aligned}
$$

That is, the equation

$$
G(\lambda)=\sum_{i=0}^{k} p_{i} e^{\lambda \tau_{i}}-\lambda^{n}=0
$$

has no roots in $[0, \infty)$. But

$$
G(0)=\sum_{i=0}^{k} p_{i}>0 \quad \text { and } \quad \lim _{\lambda \rightarrow \infty} G(\lambda)=\infty .
$$

Hence

$$
m \equiv \min _{\lambda \geq 0}\left(\sum_{i=0}^{k} p_{i} e^{\lambda \tau_{i}}-\lambda^{n}\right)
$$

exists and is a positive constant.

ProOF OF LemMa 3. From (1) we have

$$
(-1)^{n} x^{(n)}(t)=\sum_{i=0}^{k} p_{i} x\left(t-\tau_{i}\right)>0 .
$$

This implies that $x^{(n-1)}(t)$ is strictly increasing if $n$ is even or strictly decreasing if $n$ is odd. In either case $x^{(n-1)}(t)$ is eventually positive or eventually negative. Thus $(-1)^{n-1} x^{(n-1)}(t)$ is eventually either positive or negative. If $(-1)^{n-1} x^{(n-1)}(t)<0$ then

$$
(-1)^{n} x^{(n)}(t)(-1)^{n-1} x^{(n-1)}(t)<0 \quad \text { or } \quad x^{(n)}(t) x^{(n-1)}(t)>0
$$

so both $x^{(n)}(t)$ and $x^{(n-1)}(t)$ are of the same sign. Integrating $x^{(n)}(t)$ (twice) it follows that $\left|x^{(n-2)}(t)\right| \rightarrow \infty$ as $t \rightarrow \infty$ and, consequently, $|x(t)| \rightarrow \infty$ as $t \rightarrow \infty$, which contradicts the hypothesis that $x(t)$ is bounded. Therefore we have proved that eventually

$$
(-1)^{n-1} x^{(n-1)}(t)>0 .
$$


Repeating the same argument $n$ times (or using induction) the desired result follows.

Proof of Lemma 4. From (1) we have

$$
(-1)^{n} x^{(n)}(t)=\sum_{i=0}^{k} p_{i} x\left(t-\tau_{i}\right)>p_{k} x\left(t-\tau_{k}\right) .
$$

Integrating over the interval $\left[t, t+\tau_{k} /(n+1)\right]$ we find

$$
(-1)^{n} x^{(n-1)}\left(t+\frac{\tau_{k}}{n+1}\right)-(-1)^{n} x^{(n-1)}(t)>\int_{t}^{t+\left(\tau_{k} / n+1\right)} p_{k} x\left(s-\tau_{k}\right) d s .
$$

Using (4) and the fact that $x(t)$ decreases we obtain

$$
(-1)^{n-1} x^{(n-1)}(t)>p_{k}\left(\frac{\tau_{k}}{n+1}\right) x\left(t-\frac{n}{n+1} \tau_{k}\right) .
$$

Repeating the above procedure of integration over the interval $\left[t, t+\tau_{k} /(n+1)\right]$ we find (after $n$ steps)

$$
x(t)>p_{k}\left(\frac{\tau_{k}}{n+1}\right)^{n} x\left(t-\frac{1}{n+1} \tau_{k}\right), \quad \text { eventually. }
$$

Set $A=p_{k}\left(\tau_{k} /(n+1)\right)^{n}$. Then, eventually

$$
x(t)>A x\left(t-\frac{1}{n+1} \tau_{k}\right)>A^{2} x\left(t-\frac{2}{n+1} \tau_{k}\right)>\cdots>A^{n+1} x\left(t-\tau_{k}\right)
$$

and (5) holds with $B=A^{-(n+1)}$. Since $x(t)$ is strictly decreasing, $B$ has to be greater than 1.

Proof OF Lemma 5. From Taylor's Theorem there exists a point $\xi \in\left(t-\tau_{k}, t\right)$ such that

$$
x\left(t-\tau_{k}\right)=x(t)-\tau_{k} x^{\prime}(t)+\left(\tau_{k}^{2} / 2\right) x^{\prime \prime}(\xi)>x(t)-\tau_{k} x^{\prime}(t)
$$

or

$$
\tau_{k} x^{\prime}(t)+x(t)\left[x\left(t-\tau_{k}\right) / x(t)-1\right]>0
$$

Using (5),

$$
\tau_{k} x^{\prime}(t)+(B-1) x(t)>0 \text { that is, } x^{\prime}(t)+\lambda x(t)>0 .
$$

This implies that $x(t) e^{\lambda t}$ is an increasing positive function and, consequently, it cannot tend to zero.

PROOF OF LEMMA 6. (i) In view of the fact that (1) is linear with constant coefficients, the derivative of any solution of (1) is also a solution of the same equation and $x_{1}(t)$, being a linear combination of solutions of $(1)$, is itself a solution. And because of $(4), x_{1}(t)$ is eventually positive. Finally, an argument similar to that used in Lemma 3 establishes (10) for $j=1$. The proof of $(10)$ for any $j$ follows by mathematical induction.

(ii) For $j=0,(11)$ reduces to (5) with $B=B_{0}$. For any $j=1,2, \ldots$ the proof of (11) is identical to the proof given in Lemma 4.

(iii) From the definition of $x_{j}(t)$ it follows that

$$
x_{j+1}^{\prime}(t)+\lambda_{j} x_{j+1}(t)=(-1)^{n+1} x_{j}^{(n)}(t)+\lambda_{j}^{n} x_{j}(t) .
$$


Next we show, by induction, that each term in (15) is negative. In fact, for $j=0$ we have

$$
\begin{aligned}
(-1)^{n+1} x_{0}^{(n)}(t)+\lambda_{0}^{n} x_{0}(t) & =(-1)^{n+1} x^{(n)}(t)+p_{1} x(t) \\
& <(-1)^{n+1} x^{(n)}(t)+\sum_{i=0}^{k} p_{i} x\left(t-\tau_{i}\right)=0
\end{aligned}
$$

so (12) is true for $j=0$. Now assuming that (12) holds for some $j$ we should prove that it is true for $j+1$. By the inductive hypothesis, $x_{j+1}^{\prime}(t)+\lambda_{j} x_{j+1}(t)<0$ so

$$
x_{j+1}^{\prime}(t) / x_{j+1}(t)+\lambda_{j}<0 .
$$

Integrating the last inequality over $\left[t-\tau_{i}, t\right]$ we find

$$
\ln \left(x_{j+1}(t) / x_{j+1}\left(t-\tau_{i}\right)\right)+\lambda_{j} \tau_{i}<0 \quad \text { or } \quad x_{j+1}\left(t-\tau_{i}\right)>e^{\lambda_{j} \tau_{i}} x_{j+1}(t)
$$

Using the last inequality and the fact that $x_{j+1}(t)$ is a positive decreasing solution of (1), we obtain

$$
\begin{aligned}
0 & =(-1)^{n+1} x_{j+1}^{(n)}(t)+\sum_{i=0}^{k} p_{i} x_{j+1}\left(t-\tau_{i}\right) \\
& >(-1)^{n+1} x_{j+1}^{(n)}(t)+\left(\sum_{i=0}^{k} p_{i} e^{\lambda_{j} \tau_{i}}\right) x_{j+1}(t) .
\end{aligned}
$$

But from (3) and (8),

$$
\sum_{i=0}^{k} p_{i} e^{\lambda_{j} \tau_{i}} \geq \lambda_{j}^{n}+m=\lambda_{j+1}^{n}
$$

and, therefore,

$$
(-1)^{n+1} x_{j+1}^{(n)}(t)+\lambda_{j+1}^{n} x_{j+1}(t)<0 .
$$

The proof of Lemma 6 is complete.

Proof of LEMMA 7. (i) First we show that

$$
\liminf _{t \rightarrow \infty}\left[x_{j}(t) e^{\lambda_{j} t}\right]=0 .
$$

In fact, if (16) were false, then

$$
\mu \equiv \liminf _{t \rightarrow \infty}\left[x_{j}(t) e^{\lambda_{j}(t)}\right]>0 .
$$

Choose

$$
\mu_{1} \in\left(\frac{\mu}{1+m / \lambda_{j}^{n}}, \mu\right)
$$


where $m$ was defined in (3). Then by Taylor's theorem, with $t<s$, we have

$$
\begin{aligned}
x_{j}(t) & =\sum_{i=0}^{n-1} \frac{x_{j}^{(i)}(s)}{i !}(t-s)^{i}+\int_{s}^{t} \frac{(t-u)^{n-1}}{(n-1) !} x_{j}^{(n)}(u) d u \\
& =\sum_{i=0}^{n-1} \frac{(-1)^{i} x_{j}^{(i)}(s)}{i !}(s-t)^{i}+(-1)^{n} \int_{s}^{t} \frac{(t-u)^{n-1}}{(n-1) !}\left[\sum_{i=0}^{k} p_{i} x_{j}\left(u-\tau_{i}\right)\right] d u \\
& \left.>\sum_{i=0}^{k} p_{i} \int_{t}^{s} \frac{(u-t)^{n-1}}{(n-1) !} x_{j}\left(u-\tau_{i}\right) d u \quad \text { (and letting } s \rightarrow \infty\right) \\
& >\sum_{i=0}^{k} p_{i} e^{\lambda_{j} \tau_{i}} \int_{t}^{\infty} \frac{(u-t)^{n-1}}{(n-1) !} x_{j}\left(u-\tau_{i}\right) e^{\lambda_{j}\left(u-\tau_{i}\right)} e^{-\lambda_{j} u} d u \\
& \geq \mu_{1}\left(\lambda_{j}^{n}+m\right) \int_{t}^{\infty} \frac{(u-t)^{n-1}}{(n-1) !} e^{-\lambda_{j} u} d u \\
& =\mu_{1}\left(\lambda_{j}^{n}+m\right) \frac{e^{-\lambda_{j} t}}{\lambda_{j}^{n}}, \quad \text { eventually. }
\end{aligned}
$$

Hence,

$$
x_{j}(t) e^{\lambda_{j} t}>\mu_{1}\left(1+m / \lambda_{j}^{n}\right)>\mu
$$

contradicting the definition of $\mu$. Thus (16) is proved.

From (9) we have

$$
x_{j+1}(t)=\sum_{i=0}^{n-1} \lambda_{j}^{n-1-i}(-1)^{i} x_{j}^{(i)}(t)
$$

so

$$
x_{j+1}(t) e^{\lambda_{j} t}=\sum_{i=0}^{n-1} \lambda_{j}^{n-1-i}(-1)^{i}\left[x_{j}^{(i)}(t) e^{\lambda_{j} t}\right] .
$$

Also from (12) we find that $\left[x_{j+1}(t) e^{\lambda_{j} t}\right]^{\prime}<0$, therefore

$$
l \equiv \lim _{t \rightarrow \infty}\left[x_{j+1}(t) e^{\lambda_{j} t}\right]
$$

exists and is greater than or equal to zero. Since each term under the summation sign in (17) is positive, the proof of part (i) of this lemma will be complete if we show that $l=0$. To this end, using $x_{j+1}$ in place of $x_{j}$ we find (as above)

$$
\liminf _{t \rightarrow \infty}\left[x_{j+1}(t) e^{\lambda_{j} t}\right]=0
$$

so $l=0$.

(ii) From (9) we find

$$
x_{j}(t) e^{\lambda_{j} t}=\sum_{i=0}^{n-1} \lambda_{j-1}^{n-1-i}(-1)^{i} x_{j-1}^{(i)}(t) e^{\lambda_{j} t} .
$$

Since every term under the summation sign is positive, and since the limit of the left-hand side as $t \rightarrow \infty$ is zero, it follows that

$$
\lim _{t \rightarrow \infty}\left[x_{j-1}^{(i)}(t) e^{\lambda_{j} t}\right]=0 .
$$


Repeating the above argument after $j$ steps we obtain

$$
\lim _{t \rightarrow \infty}\left[x^{(i)}(t) e^{\lambda_{j} t}\right]=0,
$$

which, for $i=0$, is the desired result.

3. Remarks. The case $n=1$ was first investigated by Tramov [10] in 1975 . The same result was rediscovered in 1982 by the authors of this paper [5], by Hunt and Yorke [2] in an unpublished paper communicated to the authors, and by Arino, Györi and Jawhari $[\mathbf{1}]$. form

In the case of differential equations with one delay, that is, for equations of the

$$
x^{(n)}(t)+(-1)^{n+1} p x(t-\tau)=0, \quad p, \tau>0 ; n \geq 1,
$$

we obtain a necessary and sufficient condition in terms of the coefficient $p$ and the delay $\tau$ only. In this case the characteristic equation of $(18)$ is

$$
G(\lambda) \equiv \lambda^{n}+(-1)^{n+1} p e^{-\lambda \tau}=0 .
$$

We prove the following theorem.

THEOREM 3. For $n$ odd [ $n$ even] the following statements are equivalent.

(a) All solutions of (18) oscillate [all bounded solutions of (18) oscillate].

(b) Equation (19) has no real roots [(19) has no real roots in $(-\infty, 0]]$.

(c) $p^{1 / n} \tau / n>1 / e$.

An analogue to Lemma 1 also holds for (18). Therefore, the proof of Theorem 3 is reduced to proving the following result for arbitrary $n$.

THEOREM 4. The following statements are equivalent.

(a) Every bounded solution of (18) oscillates.

(b) The characteristic equation (19) has no real roots in $(-\infty, 0]$.

(c) $p^{1 / n} \tau / n>1 / e$.

ProOF. First we prove (a) implies (b). Otherwise there exists a real $\lambda_{0} \leq 0$ such that

$$
\lambda_{0}^{n}+(-1)^{n+1} p e^{-\lambda_{0} \tau}=0,
$$

which implies that $x(t)=e^{\lambda_{0} t}$ is a nonoscillatory solution of (18), a contradiction.

Next we prove (b) implies (c). Equation (19) is equivalent to

$$
G(\lambda) \equiv(-1)^{n} \lambda^{n}-p e^{-\lambda \tau}=0 .
$$

Since $G(0)=-p<0$ and $G(\lambda)$ has no negative roots, it follows that $G(\lambda)<0$ for every $\lambda \in(-\infty, 0]$. Setting $\lambda=-n / \tau$ into $G(\lambda)$ we find

$$
(-1)^{n}\left(-\frac{n}{\tau}\right)^{n}-p e^{n}<0 \Rightarrow p e^{n}>\left(\frac{n}{\tau}\right)^{n} \Rightarrow p^{1 / n} \frac{\tau}{n}>\frac{1}{e} .
$$

Finally we prove (c) implies (a). Otherwise (18) has an eventually positive bounded solution $x(t)$. As in Lemma 3 , for $i=0,1 \ldots, n$,

$$
(-1)^{i} x^{(i)}(t)>0, \quad \text { eventually. }
$$


Set $q=p^{1 / n}, \sigma=\tau / n$ and

$$
\begin{aligned}
y(t)= & \sum_{j=0}^{n-1}(-1)^{j} q^{j} x^{(n-1-j)}(t-j \sigma) \\
= & x^{(n-1)}(t)-q x^{(n-2)}(t-\sigma)+q^{2} x^{(n-3)}(t-2 \sigma) \\
& -\cdots+(-1)^{n-1} q^{n-1} x(t-(n-1) \sigma) .
\end{aligned}
$$

Then, in view of (4),

$$
(-1)^{n+1} y(t)>0, \quad \text { eventually. }
$$

Observe that

$$
\begin{aligned}
y^{\prime}(t)= & \sum_{j=0}^{n-1}(-1)^{j} q^{j} x^{(n-j)}(t-j \sigma) \\
= & x^{(n)}(t)-q x^{(n-1)}(t-\sigma)+q^{2} x^{(n-2)}(t-2 \sigma) \\
& -\cdots+(-1)^{n-1} q^{n-1} x^{\prime}(t-(n-1) \sigma)
\end{aligned}
$$

and, therefore,

$$
y^{\prime}(t)+q y(t-\sigma)=x^{(n)}(t)+(-1)^{n+1} p x(t-\tau)=0 .
$$

But the characteristic equation of

$$
y^{\prime}(t)+q y(t-\sigma)=0
$$

is

$$
\lambda+q e^{-\lambda \sigma}=0
$$

and, in view of $(c)$,

$$
\min _{\lambda}\left(\lambda+q e^{-\lambda \sigma}\right)=\frac{1}{\sigma} \ln (q \sigma e)=\frac{1}{\sigma} \ln \left(p^{1 / n} \frac{\tau}{n} e\right)>0 .
$$

That is, (22) has no real roots and therefore (see [5]) every solution $y(t)$ of $(21)$ is oscillatory. This contradicts (20).

The proof of the theorem is complete.

The above results have straightforward extensions to differential equations with advanced arguments of the forms

$$
x^{(n)}(t)-\sum_{i=0}^{k} p_{i} x\left(t+\tau_{i}\right)=0
$$

and

$$
x^{(n)}(t)-p x(t+\tau)=0 .
$$

The characteristic equations of $(1)^{\prime}$ and $(18)^{\prime}$ are, respectively,

$$
f(\lambda) \equiv \lambda^{n}-\sum_{i=0}^{k} p_{i} e^{\lambda \tau_{i}}=0
$$

and

$$
g(\lambda) \equiv \lambda^{n}-p e^{\lambda \tau}=0 .
$$

By similar arguments we establish the following dual results. 
THEOREM $1^{\prime}$. (i) For $n$ odd all solutions of $\left(1^{\prime}\right)$ oscillate if and only if $(2)^{\prime}$ has no real roots.

(ii) For $n$ even all unbounded solutions of $(1)^{\prime}$ oscillate if and only if (2)' has no real roots in $[0, \infty)$.

THEOREM $3{ }^{\prime}$. For $n$ odd [ $n$ even] the following statements are equivalent.

(a) All solutions of $(18)^{\prime}$ oscillate [all unbounded solutions of (18)' oscillate].

(b) Equation $(19)^{\prime}$ has no real roots $\left[(19)^{\prime}\right.$ has no real roots in $\left.[0, \infty)\right]$.

(c) $p^{1 / n} \tau / n>1 / e$.

\section{REFERENCES}

1. O. Arino, I. Györi and A. Jawhari, Oscillation criteria in delay equations, J. Differential Equations (to appear).

2. B. R. Hunt and J. A. Yorke, When all solutions of $x^{\prime}=-\sum_{i=1}^{n} q_{i}(t) x\left(t-T_{i}(t)\right)$ oscillate (preprint).

3. T. Kusano, On even onder functional differential equations with advanced and retarded arguments, J. Differential Equations 45 (1982), 75-84.

4. G. Ladas, Sharp conditions for oscillations caused by delays, Applicable Anal. 9 (1979), 93-98.

5. G. Ladas, Y. G. Sficas and I. P. Stavroulakis, Necessary and sufficient conditions for oscillations, Amer. Math. Monthly 90 (1983), 637-640.

6. G. Ladas and I. P. Stavroulakis, On delay differential inequalities of higher order, Canad. Math. Bull. 25 (1982), 348-354.

7. __ Oscillations caused by several retarded and advanced arguments, J. Differential Equations 44 (1982), 134-152.

8. V. N. Sevelo and N. V. Vareh, Asymptotic methods in the theory of nonlinear oscillations, "Naukova Dumka", Kiev, 1979. (Russian)

9. Y. G. Sficas and V. A. Staikos, Oscillations of differential equations with deviating anguments, Funkcial. Ekvac. 19 (1976), 35-43.

10. M. I. Tramov, Conditions for oscillatory solutions of first order differential equations with a delayed argument, Izv. Vysš. Ucebn. Zaved. Matematika 19 (1975), 92-96.

DEPARTMENT OF MATHEMATICS, UNIVERSity OF RHODE ISLAND, Kingston, RHOdE ISLAND 02881 (Current address of G. Ladas)

Department of Mathematics, Univeristy of IoAnNina, Ioannina, Greece (Current address of Y. G. Sficas and I. P. Stavroulakis) 